\title{
CONOCIMIENTO DE LA LEY GENERAL DE SALUD RESPECTO DE LAS TRANSFUSIONES SANGUÍNEAS EN MÉDICOS Y PACIENTES TESTIGOS DE JEHOVÁ DEL HOSPITAL DR. DARÍO CONTRERAS DE REPÚBLICA DOMINICANA
}

Elsa Díaz Santana*

\begin{abstract}
Resumen: Este estudio evalúa hasta qué grado el cuerpo médico del Hospital Dr. Darío Contreras de República Dominicana conoce, respeta, informa y aplica la Ley General de Salud, con relación al derecho del paciente Testigo de Jehová de negarse a ser transfundido (respeto a su autonomía). También si los Testigos de Jehová conocen la Ley General de Salud y hasta qué grado se han beneficiado con la puesta en marcha de la misma. El estudio reveló que ni médicos ni Testigos de Jehová conocen suficientemente dicha ley.
\end{abstract}

Palabras clave: Ley General de Salud, transfusiones sanguíneas, Testigos de Jehová, autonomía

\section{KNOWLEDGE OF GENERAL HEALTH LAW WITH RESPECT TO BLOOD TRANSFUSIONS BY PHYSICIANS AND PATIENTS JEHOVAH WITNESSES IN HOSPITAL DR. DARIO CONTRERAS OF DOMINICAN REPUBLIC}

Abstract: This study evaluates up to which degree physicians of Hospital Dr. Dario Contreras of Dominican Republic know, respect, inform and apply the General Health Law in relation to the right of Jehovah witness patients to refuse being blood transfused (respect to their autonomy). It also evaluates whether Jehovah witnesses know General Health Law and in which degree they have been benefited by putting it into practice. The study reveals that Jehovah Witnesses do not know the law.

Key words: General Health Law, blood transfusions, Jehovah Witnesses, autonomy

\section{CONHECIMENTO DA LEI GERAL DE SAÚDE - RESPEITO ÀS TRANSFUSÓES SANGUÍNEAS EM MÉDICOS E PACIENTES TESTEMUNHAS DE JEOVÁ DO HOSPITAL DR. DARÍO CONTRERAS DA REPÚBLICA DOMINICANA}

Resumo: Este estudo avalia quanto o corpo médico do Hospital Dr. Darío Contreras de República Dominicana conhece, respeita, informa e aplica a Lei Geral de Saúde em relação aos direitos do paciente Testemunha de Jeová de negar-se a ser transfundido (respeito a sua autonomia); também se os Testemunhas de Jeová conhecem a Lei Geral de Saúde e até que ponto têm se beneficiado diante dessa proposição. $\mathrm{O}$ estudo revelou que nem médicos, nem Testemunhas de Jeová conhecem de fato essa lei.

Palavras-chave: Lei Geral de Saúde, transfusões sanguíneas, Testemunhas de Jeová, autonomia

\footnotetext{
Farmacéutica. Jefe de Servicio de Farmacia. Vicepresidenta del Comité de Ética y de Investigación del Centro de Diagnóstico y Medicina Avanzada y de Conferencias Médicas y de Telemedicina (CEDIMAT), Santo Domingo, República Dominicana

Becaria del Programa de Ética de la Investigación Biomédica y Psicosocial, Fogarty Internacional Center, Grant R25 TW006056, 2009.

Correspondencia: elsadiaz2001@yahoo.com
} 


\section{Introducción}

El marco jurídico del sistema sanitario de la República Dominicana cambió de manera significativa con la promulgación, en 2001, de la Ley General de Salud (42-01), en la cual a la Secretaría de Estado de Salud Pública y Asistencia Social se le confiere la función rectora, estando obligada a redefinir y readecuar sus estructuras funcionales.

El capítulo IV, art. 28 de la Ley General de Salud establece el respeto a la dignidad humana y a no ser discriminado por razones religiosas, además del derecho del paciente a decidir, previa información.

Existe el Código del Menor, cuyo objeto es garantizar a nińos y adolescentes que se encuentren en el territorio nacional el ejercicio y disfrute pleno y efectivo de sus derechos fundamentales. En el caso de hijos de padres Testigos de Jehová, quienes no aceptan transfusión sanguínea, prima la beneficencia desde el punto de vista del médico, siempre y cuando peligre la vida del menor.

En los años 90, los Testigos de Jehová habían sido objeto de discriminación al rehusar ser transfundidos. Entienden ellos que las transfusiones sanguíneas pueden añadir años a su vida, pero no salvan la vida para siempre.

Este estudio evalúa hasta qué grado el médico conoce, respeta, informa y aplica la Ley General de Salud con relación al derecho del paciente Testigo de Jehová a negarse a ser transfundido (respeto a su autonomía). También si los Testigos de Jehová conocen la Ley General de Salud y hasta qué grado se han beneficiado con su puesta en marcha.

\section{Materiales y métodos}

Estudio descriptivo cualitativo, en el cual se investigaron las siguientes variables:

- Nivel de conocimiento de los médicos sobre la Ley General de Salud y aplicación de la misma.

- Nivel de conocimiento de los Testigos de Jehová sobre la Ley General de Salud.

- Problemas de factibilidad de cumplimiento de dicha Ley.

\section{Muestra}

En octubre de 2008 se encuestó a 26 médicos de ambos sexos del hospital Dr. Darío Contreras, Santo Domin- go, República Dominicana, de las especialidades de cirugía general $31 \%$, emergentología $23 \%$, ortopedia $23 \%$, neurocirugía $11 \%$, urología $8 \%$ y gastroenterología $4 \%$, con edades que fluctuaban entre 26 y 64 años, $58 \%$ del sexo masculino y $42 \%$ del sexo femenino.

Por otra parte, se encuestó a 16 pacientes Testigos de Jehová, con edades entre 18 y 60 años, $75 \%$ del sexo femenino y el $25 \%$ del sexo masculino.

\section{Instrumentos}

- Análisis de la Ley General de Salud en sus aspectos bioéticos.

- Reflexión sobre el derecho de autonomía de los Testigos de Jehová en relación con sus creencias sobre las transfusiones de sangre.

Se elaboraron dos cuestionarios de preguntas abiertas: uno para los médicos y otro para los Testigos de Jehová.

A los médicos se les aplicó un cuestionario de 18 preguntas sobre el conocimiento de la Ley General de Salud y su aplicación, el consentimiento informado, el respeto a la autonomía del paciente y la decisión del médico. Una pregunta se refería a la decisión del médico de transfundir a un menor, respetar la voluntad de los padres o llevar el caso a la justicia.

Las preguntas incluyeron aclaraciones del tipo: "por qué y explique".

El cuestionario a Testigos de Jehová reunía un total de 12 preguntas abiertas sobre el conocimiento de la Ley General de Salud, el trato recibido una vez hospitalizados, la hospitalización de un hijo menor y la necesidad de transfusión y el consentimiento informado y el respeto a la autonomía.

\section{Otros antecedentes}

\section{Ley General de Salud}

En la República Dominicana, el siglo XXI se inició con la gran apuesta de transformar, en un plazo de diez años, todo el sistema de salud del país, empezando por redefinir incluso lo que se entiende por salud y cómo debería llevarse a cabo ese cambio, ya que existía el viejo Código de Trujillo, vigente desde 1956. A dos meses de su puesta en marcha se creó la Ley del Sistema Dominicano de Seguridad Social (87-01)(1). Estas 
dos nuevas leyes introducían cambios verdaderamente radicales en lo que hasta ese momento existía, cambios que debían ser "graduales y progresivos(2)".

Aunque la Ley General de Salud no especifica la no transfusión, sí exige respeto al derecho de autonomía del paciente. El artículo 11 del capítulo II, sección II, y el artículo 3 del capítulo I establecen que el Sistema Nacional de Salud se regirá por los principios de universalidad, solidaridad, equidad, eficiencia, eficacia, integridad y cooperación. Desde estos principios, el Estado debe garantizar un nivel mínimo de prestaciones.

Callahan (1998) señala que "...el principio de solidaridad constituye uno de los principales ingredientes para pensar en una medicina que sea al mismo tiempo equitativa y sustentable" (3). Sin embargo, el principio de solidaridad se expone a innumerables críticas, sobre todo si se utiliza para legitimar políticas de asignación de recursos, los cuales son reconocidamente finitos y escasos en cualquier sistema sanitario.

La escasez de los recursos asignados al sector salud no ha permitido dar una educación continua a los profesionales, la infraestructura en los centros hospitalarios está obsoleta y deteriorada y la reconstrucción sobre la misma ha empeorado la situación. En cuanto a la tecnología, se han asignado bastantes recursos en equipos sofisticados, pero el personal en general no sabe manejarlos y se han dañado con el correr de los años; es decir, impera la inequidad, ineficiencia, ineficacia e injusticia, ya que no siempre existen las condiciones para satisfacer las necesidades de salud de los pacientes. En tales circunstancias, los pacientes Testigos de Jehová no siempre pueden exigir su derecho a la autonomía y ofrecer métodos alternativos a una transfusión sanguínea.

Aunque existen estudios que reflejan un impacto positivo de la reforma del sector salud(4), la verdad es que en conocimientos, capacitación o educación se ha avanzado a pasos muy lentos. A mayor conocimiento y mejor educación y capacitación la información al paciente será más efectiva y habrá mayor respeto por la autonomía del paciente.

El capítulo IV de la Ley General de Salud(2) se refiere a los derechos y deberes con relación a la salud. El artículo 28 establece lo siguiente:

- Toda persona tiene derecho al respeto a su personalidad, dignidad humana e intimidad, a no ser discriminada por razones de etnia, edad, religión, condición social, política, sexo, estado legal, situa- ción económica, limitaciones físicas, intelectuales, sensoriales o cualquier otra.

- A la educación y prevención de las enfermedades y a la conservación y recuperación de la salud.

- A la información sobre los servicios que promuevan y protejan la salud, al acceso a los mismos y a una atención adecuada y oportuna.

- A la confidencialidad de todas las informaciones relacionadas con su expediente.

- A la información adecuada y continuada de su proceso, incluyendo su diagnóstico, pronóstico y alternativas de tratamiento, a recibir consejos por el personal capacitado, antes y después de la realización de exámenes y procedimientos.

- $\quad \mathrm{Al}$ derecho a decidir previa información y comprensión sobre su aceptación o rechazo de asumir el tratamiento. Se exceptúan de estas disposiciones los casos que representan riesgos para la salud pública.

- En el caso de menores, discapacitados mentales y pacientes en estado crítico, sin conciencia para decidir, la decisión recae sobre los familiares directos, tutores o, en ausencia, sobre el médico principal responsable de su atención.

- Al derecho a no ser sometido/a a tratamiento médico o quirúrgico que implique grave riesgo para su integridad física, su salud o su vida, sin su consentimiento escrito o el de la persona responsable. Esto último sólo en el caso de que el paciente no esté en capacidad para darlo y siempre que sea en su beneficio.

Si se ofrece información oportuna clara y eficaz a los pacientes sobre su diagnóstico, cirugía o tratamiento, riesgos y beneficios, éstos pueden decidir sobre lo que consideran más adecuado. En el caso de que el médico considere que deban ser transfundidos, los pacientes Testigos de Jehová pueden ofrecer otras alternativas que no violen sus principios y creencias ${ }^{1}$. De esta manera, se pone de manifiesto el consentimiento informado y el respeto a la autonomía del paciente.

1 "Los Testigos de Jehová consideran fundamental que sus creencias estén basadas en la Biblia y no en simples conjeturas o credos religiosos de hombres. Creen que toda enseñanza religiosa -la ofrezcan ellos u otras personas- debe someterse a esta prueba de concordancia con las Escrituras inspiradas por Dios. 'Testigos de Jehová' es un nombre descriptivo que indica que dan testimonio de Jehová, su divinidad y sus propósitos". Una descripción más completa de lo citado en: ¿Cómo puede salvarle la vida la sangre? Watch Tower Bible and Tract Society of Pennsylvania: 2005. Disponible en http://www.watchtower.org/s/ $\mathrm{hb} /$ index.htm?article=article_00.htm 
Aunque no se está cumpliendo por completo, la realidad es que, luego de implementada la ley, se brinda un mayor respeto a los pacientes Testigos de Jehová que en los años anteriores a la puesta en vigencia de dicha Ley.

Una de las partes más importante de la ley es garantizar el derecho del paciente a la información comprensible y veraz. Por formación, el médico es paternalista; sin embargo, mediante esta Ley el médico está obligado a informar al paciente con palabras claras sobre riesgos y beneficios para que este tome la decisión.

\section{Desafío quirúrgico/ético}

Los médicos se enfrentan a un desafío creciente que es una importante cuestión de salud. En Estados Unidos hay más de medio millón de Testigos de Jehová y ellos no aceptan transfusiones de sangre. La cantidad de Testigos y de los que se asocian con ellos está aumentando. Aunque antes, cuando alguien rehusaba una transfusión, muchos médicos y ejecutivos de los hospitales veían esto como un problema legal y procuraban la autoridad de los tribunales para proceder, literatura médica publicada recientemente revela que está aconteciendo un notable cambio de actitudes. "Puede que esto sea el resultado de más experiencia quirúrgica con pacientes cuyo nivel de hemoglobina es muy bajo, y puede que también refleje mayor reconocimiento del principio legal del consentimiento informado" 2 .

Ahora, en muchos casos de cirugía electiva y de traumatismo, en que figuran tanto Testigos adultos como menores, se está logrando el objetivo deseado sin transfusiones de sangre. Hace poco, ciertos representantes de los Testigos de Jehová se reunieron con miembros del personal quirúrgico y administrativo de algunos de los centros médicos más grandes de Puerto Rico. Estas reuniones mejoraron la comunicación y comprensión y ayudaron a resolver cuestiones acerca del rescate sanguíneo, los trasplantes y la evitación de confrontaciones médico-legales.

\section{Resultados de la encuesta}

El 38\% de los médicos afirmó no conocer la Ley General de Salud, por tanto, sólo el 62\% dijo conocerla. $\mathrm{Al}$ inquirir sobre los artículos que protegen la toma

2 Los Testigos de Jehová: el desafío quirúrgico/ético. En: ¿Cómo puede salvarle la vida la sangre? Watch Tower Bible and Tract Society of Pennsylvania: 2005. Disponible en: http://www.watchtower.org/s/ $\mathrm{hb} /$ index.htm?article=article_01.htm de decisiones de parte del paciente y pedir explicar, el $46 \%$ de los médicos dijo no saber, el 54\% no contestó y ninguno dio explicación, lo que indica la gran necesidad de información sobre la Ley General de Salud, especialmente sobre el artículo 28 del capítulo IV que trata de los deberes y derechos del paciente.

A la pregunta sobre si conocía la Ley, el $81 \%$ de los Testigos de Jehová encuestados respondió que no la conocía, pero cuando se les preguntó al 19\% restante sobre los artículos que conoce de ella, el 43,75\% citó el Libro de los Hechos, capítulo 15, versículo 28, en el cual La Biblia ordena abstenerse de sangre. Los Testigos de Jehová tienen claridad, entonces, acerca de los principios bíblicos que prohíben ingerir sangre, no así sobre la información contenida en la Ley General de Salud respecto de estas situaciones.

Acerca de si tenían conocimiento de algún artículo de la Ley sobre la no transfusión, un $85,71 \%$ de los Testigos de Jehová respondieron que no. Al preguntar al 14,29\% restante acerca del contenido de la ley, el $100 \%$ no contestó.

Con relación a si reciben habitualmente información sobre su tratamiento, cirugía o diagnóstico, el 86\% de los Testigos de Jehová respondió que sí la recibe. Lo que habrá que averiguar en estudios futuros es qué tan clara e inteligible es esta información.

\section{Discusión}

La Ley General de Salud está vigente desde 2001. Los médicos encuestados, pese a que la mayoría de ellos afirmó conocerla, no saben cuáles artículos protegen la toma de decisiones de los pacientes.

La mejora en la calidad de los servicios y la toma de decisiones consensuadas ha sido evidente a partir de la puesta en marcha de la Ley General de Salud, pero sólo en comparación con los años anteriores a la puesta en vigencia de dicha Ley.

Aunque la Ley protege a los menores en caso de que su vida peligre, cediendo el derecho al médico en la toma de decisiones beneficentes que contribuyan a salvar la vida del menor, los médicos dijeron respetar la autonomía de los padres de los menores y tomar en cuenta sus alternativas. No obstante, en el país se están produciendo irregularidades, en las cuales la fiscalía autoriza la transfusión sin una orden del juez. 
El 96\% de los médicos informa al paciente sobre riesgos y beneficios del tratamiento o cirugía, aunque sería importante investigar si lo hacen de manera clara y entendible.

El contenido del capítulo IV de la Ley General de Salud ha tenido un gran impacto, pues los médicos saben que deben respetar la dignidad del paciente, dar información adecuada, clara y entendible, como también respetar el derecho del paciente a decidir, previa comprensión de la misma.

El 92\% de los médicos señala que daría atención a pacientes testigos de Jehová, lo que indica menos discriminación que antaño y que los principios fundamentales de la bioética están llegando a los médicos. Aunque hace falta más información, más difusión de las leyes, el paternalismo está disminuyendo.

\section{Conclusión}

De acuerdo con los resultados, podemos observar el relativo impacto de la implementación de la Ley Ge- neral de Salud respecto de transfusiones sanguíneas en pacientes Testigos de Jehová.

Existe la necesidad de que se difunda y aplique la Ley General de Salud, que se apliquen sanciones a los que no la cumplen, sobre todo cuando se viola el respeto a la dignidad humana y la autonomía del paciente a decidir sobre su tratamiento o cirugía.

Para que la puesta en marcha de la Ley General de Salud sea efectiva, es preciso que las autoridades velen por su fiel cumplimiento y que propicien y proporcionen las condiciones para su puesta en marcha. Es necesario también que la población en general la conozca.

Este estudio puede servir de base para realizar otros similares, por ejemplo, en la atención directa del paciente.

\section{Agradecimientos}

Al Programa de Ética de la Investigación Biomédica y Psicosocial, Fogarty Internacional Center, Grant R25 TW006056, 2009, Centro Interdisciplinario de Estudios en Bioética, Universidad de Chile.

\section{Referencias}

1. Rathe M. La Seguridad Social. Santo Domingo, República Dominicana: Puccmma-Pid.Re- USAID; 2001.

2. Ley General de Salud (42-01) Capítulo II, Sección I y II. República Dominicana: Centro de Información y Documentación para el Desarrollo. Disponible en http://rsta.pucmm.edu.do/biblioteca/bvds/saludley.htm

3. Schramm FR, Kottow M. Principios bioéticos en salud pública. Cadernos de Saúde Pública 2001; 17(4): 949-956.

4. Del Conde A. Una Reforma Para el Sector Salud. Clave para profesionales del Sector Salud. República Dominicana: Programa de Apoyo a la Reforma del Sistema de Salud (PROSISA); 2004: 26-33.

Recibido: 20 de enero de 2010

Aceptado: 13 de abril de 2010 